\title{
Analysis of Bistatic Radar Sea Clutter Amplitude Distributions at Low Grazing Angles
}

\author{
Sébastien Angelliaume \\ DEMR, ONERA, France \\ Sebastien.Angelliaume@onera.fr
}

\author{
Luke Rosenberg \\ DST Group, Australia \\ Luke.Rosenberg@dst.defence.gov.au
}

\author{
Matthew Ritchie \\ University College London, UK \\ m.ritchie@ucl.ac.uk
}

\begin{abstract}
Target detection in the maritime domain is best performed with radar due to its ability to surveil wide areas and operate in almost any weather condition or time of day. Many common detection schemes require an accurate model of the amplitude distribution of radar echoes backscattered by the ocean surface. The paper presents quantitative evaluation of a series of selected amplitude distributions in their ability to represent real low grazing angle sea clutter collected by a ground-based bistatic radar system operating at S-band.
\end{abstract}

Keywords-radar, sea clutter, statistical analysis, amplitude distribution, K, Pareto, Rayleigh, 3MD.

\section{INTRODUCTION}

Radar sensors are powerful means for detecting targets in the maritime environment as they can be used at any time of day and in almost any weather condition. In the maritime environment, they are typically operated at low grazing angles to enhance the ratio of the target to clutter power. However, sea clutter collected from this geometry also contains many "sea-spikes" which manifest as undesired strong returns and are often the cause of false detections [1].

Over recent years, bistatic radars have received considerable attention for a number of reasons, including the extra degrees of freedom that can be used to extract information on targets and the covertness of the receive node [2]. However, bistatic measurements are significantly more difficult than monostatic ones, as there are more challenging logistics and a greater number of variables which influence the sea clutter statistics.

Target detection is typically approached with a constant false alarm rate (CFAR) scheme [1] and the selection of a threshold which limits the number of false alarms, while maintaining a desired probability of detection. This can be achieved by fitting a theoretical amplitude distribution or probability density function (PDF) to the target free radar backscatter. There has been a long history of developing PDF models used to fit experimental radar measurements. With coarse range resolution, an effective model for the sea-clutter PDF has been shown to be the Rayleigh distribution [3]. As the range resolution becomes finer, the effect of sea-spikes becomes more pronounced, especially when considering horizontally $(\mathrm{H})$ polarized waves. From a statistical point-ofview, sea-spikes produce a non-Gaussian heavy-tailed distribution [3]. A widely used framework for developing PDF models is the compound Gaussian model which was originally proposed for use in sea-clutter by Ward [4]. This model includes a temporal or fast varying component known as speckle which relates to the Bragg scattering, and a slowly varying component which captures the underlying swell and models the texture. The most popular compound model in the literature is the $\mathrm{K}$ distribution [4], [5] and its extended version which accounts for the radar instrument noise, namely the K+Noise [4]. Similarly, the Pareto distribution [6] and its extended version, the Pareto+Noise [7], have become popular recently due to trhere ability to model the extended tail of the amplitude distribution.

A relevant study into the amplitude statistics of monostatic and bistatic sea-clutter was given in [8] where the log-normal, Weibull, Pareto, K+Noise and KA distributions were studied. This paper builds on that work by studying the same NetRAD mono-static and bistatic dataset with two newer proposed sea clutter distributions from the literature: the $\mathrm{K}+$ Rayleigh [9] and the tri-modal (3MD) distributions [10][11]. The latter distribution has demonstrated great potential for modelling sea-clutter for both real aperture and synthetic aperture radar and is unique in the way it models the sea clutter texture as a combination of discrete components. Two metrics are then used to quantitatively evaluate the ability for each model to represent the actual sea clutter amplitude distribution.

This paper is organized as follows: Section II describes the goodness of fit metrics, Section III summarizes the theoretical distributions and Section IV describes the NetRAD data set. Section V then summarizes the results and concludes by identifying the most suitable distribution to model the sea clutter.

\section{MEASURES OF GOODNESS OF FIT}

This paper report on a quantitative evaluation on different PDF models for modelling radar sea clutter. Two goodness of fit measures are used: the Bhattacharyya distance (BD) and the threshold error.

\section{A. The Bhattacharyya Distance}

The Bhattacharyya distance is a metric which varies between 0 and infinity. It measures the similarity between the theoretical PDF P(.) and the actual data distribution $\mathrm{Q}($.$) . The$ smaller the Bhattacharyya distance, the better the goodness of fit and thus the accuracy of the model to fit the radar data [5].

$$
B D=-\ln \left(\sum_{x_{k}} \sqrt{P\left(x_{k}\right) Q\left(x_{k}\right)}\right) \quad 0 \leq B D \leq \infty
$$




\section{B. The Threshold Error}

The second metric is the threshold error which is determined by first calculating the complementary cumulative distribution function (CCDF) for both the empirical data and the model. It is then the difference between the two results at a fixed CCDF value, often $10^{-4}$ or lower, depending on operational requirements [12]. The CCDF is important due to its relationship with the threshold in a detection scheme used for distinguishing between targets and interference.

\section{AMPLITUDE DISTRIBUTION MODELS}

In target detection analysis, the envelope of the received pulses is often converted to power (square law) and the clutter distribution becomes exponential. For a frequency agile or scanning radar with sufficient time between looks, a common method to improve the detection performance is to sum a number of looks. If there are $M$ independent exponential random variables, $\mathrm{z}=\sum_{\mathrm{m}=1}^{\mathrm{M}} \mathrm{y}_{\mathrm{m}}$, then the received power is described by a gamma PDF,

$$
P(z \mid x)=\frac{z^{M-1}}{\left(x+\sigma_{n}^{2}\right)^{M} \Gamma(M)} \exp \left[-\frac{z}{x+\sigma_{n}^{2}}\right]
$$

where $0 \leq \mathrm{x} \leq \infty, \Gamma$ (.) is the gamma function and $\sigma_{n}^{2}$ is the instrument noise power. In order to include the texture component which modulates the speckle, we integrate over the speckle mean power,

$$
P(z)=\int_{0}^{\infty} P(z \mid x) P(x) d x
$$

where $P(x)$ is the distribution of the texture component. While there are analytic solutions in many cases, when instrument noise is included in the model, numerical integration must be used to evaluate the compound distribution. The parameters of the $\mathrm{K}+$ Noise, Pareto+Noise and $\mathrm{K}+$ Rayleigh distributions evaluated herein are estimated using the zlogz method which has demonstrated the best tradeoff for accuracy and computational efficiency [13]. However for the 3MD distribution, there is no suitable zlogz estimator and the model parameters are estimated using a least squares minimization between the CCDF of the data and the model. Also, in the following, only single-look intensity data is considered with the data represented in decibels $(\mathrm{dBs})$.

\section{A. The K+Noise Distribution}

The $\mathrm{K}+$ Noise $(\mathrm{KN})$ distribution is a well-established model for representing high-resolution radar sea clutter. It is a continuous mixture of an exponential or gamma distribution for the uncorrelated speckle intensity (fast temporal variation) with a gamma distribution for the clutter power (slow spatial variation) [4].

$$
P(x)=\frac{b^{v}}{\Gamma(v)} x^{v-1} \exp [-b x]
$$

where $v>0$ and $b=v /\langle x\rangle$ are the shape and scale parameters respectively and $\langle x\rangle$ is the mean power.

\section{B. The Pareto +Noise Distribution}

The Pareto+Noise (PN) distribution is another popular compound distribution used to model the sea-clutter backscatter [8]. It is formed with an inverse gamma distribution for the texture

$$
P(x)=\frac{d^{a}}{\Gamma(a)} x^{-a-1} \exp [-d / x]
$$

where $a$ and $d=\langle\mathrm{x}\rangle(a-1)$ are the shape and scale parameters respectively

\section{The K+Rayleigh Distribution}

The $\mathrm{K}+$ Rayleigh $(\mathrm{KR})$ distribution is an extension to the $\mathrm{KN}$ model designed to capture any extra Rayleigh component in the data which arises from the non-Bragg scattering [14]. It explicitly separates the speckle mean level into two components, $x=x_{r}+p_{r}$, where $p_{r}$ is the power of the extra Rayleigh component. As with the KN model, the texture is given by a gamma distribution

$$
P\left(x_{r}\right)=\frac{b_{r}^{v_{r}}}{\Gamma\left(v_{r}\right)} x_{r}^{v_{r}-1} \exp \left[-b x_{r}\right]
$$

where $v_{r}>0$ and $b_{r}=v_{r} /\langle x\rangle$ are the shape and scale parameters respectively. The influence of the extra Rayleigh component can be measured by the ratio of the mean of the Rayleigh component, $p_{r}$, to the mean of the gamma distributed component, $p_{c}$, of the clutter and is defined by

$$
k_{r}=\frac{p_{r}}{p_{c}}
$$

\section{The Tri-Modal Discrete Distribution}

The compound models presented previously all assume a continuous texture distribution which suggests a small probability of infinite texture values. The motivation of the 3MD model is to instead use a discrete texture model that assumes the sea clutter consists of a finite number, I, of distinct modes or scatterer types [10]. This implies that the scatterers in the observed scene are realizations from homogeneous clutter random variables with different texture values. The PDF of the texture is given by

$$
\begin{gathered}
P(x)=\sum_{n=1}^{I} c_{n} \delta\left(x-a_{n}\right), \quad \sum_{n=1}^{I} c_{n}=1, a_{n}, c_{n}>0 \\
\delta(A):= \begin{cases}1 & \text { if } A=0 \\
0 & \text { otherwise }\end{cases}
\end{gathered}
$$

where $a=\left[a_{1}, \ldots, a_{I}\right]$ are the discrete intensity texture levels and $c=\left[c_{1}, \ldots, c_{I}\right]$ are the corresponding weightings.

The 3MD distribution also requires the non-trivial estimation of $2 I$ unknown parameters. This can be achieved with a least squares minimization between the 3MD model and data CCDF in the log domain. The fitting process first assumes a single mode $(I=1)$. If the $\mathrm{BD}$ of that fit is greater than $-30 \mathrm{~dB}$, the parameters for a bi-modal fit $(I=2)$ are then estimated. This 
is repeated up to the maximum number of modes, which is set to $I=5$ unless otherwise stated. The model components, $a_{n}$, are then ordered from largest to smallest and any modes where the weightings $c_{n}<10^{-3}$ are removed. Note that the threshold value of $\mathrm{BD}=-30 \mathrm{~dB}$ comes from experimental observation where there is little change in the $3 \mathrm{MD}$ distribution.

\section{RADAR SEA ClUTTER DATASET}

The Netted Radar (NetRAD) is a ground-based coherent bistatic radar system operating at S-band (carrier frequency of $2.45 \mathrm{GHz}$ ) [15]. The system was developed jointly by the University College London, UK and the University of Cape Town, South Africa. It works in both monostatic and bistatic configurations with the two nodes synchronized in time with GPS disciplined oscillators, and the radars are networked with a $5 \mathrm{GHz}$ wireless link. The system configuration used for gathering the data analyzed here had a peak transmit power of $57.7 \mathrm{dBm}$, pulse repetition frequency (PRF) of $1 \mathrm{kHz}$, antenna gain of $23.8 \mathrm{dBi}$ and a bandwidth of $45 \mathrm{MHz}$ providing a range resolution of $3.3 \mathrm{~m}$.

The antennas work with either vertical (V) or horizontal polarizations $(\mathrm{H})$. The data is summarized in Table 1 and was obtained during a 2010 series of trials in South Africa near the Cape Point area. The system was configured to transmit and receive from one monostatic site, while simultaneously coherently measuring returns from a separate bistatic location. The baselines between each of two radar nodes and the illuminated area were $1830 \mathrm{~m}$ and in order to vary the bistatic angle, the antennas were rotated to cover different regions of the sea. The grazing angles from the geometries varied from $0.6^{\circ}$ to $1.5^{\circ}$ [15], which will have a minor impact on the backscattered signal compared to changes with the polarization or the bistatic angle. In the following analysis, 10 $s$ of data have been used for each run, resulting in more than $10^{6}$ samples. Wind and wave information was obtained from a nearby wavebuoy and is given in Table I.

TABLE I. Properties OF THE NETRAD SEA ClutTER ACQUiSitions

\begin{tabular}{ccccccc}
\hline \hline Run & Pol. & $\begin{array}{c}\text { Bistatic } \\
\text { angle } \\
\left({ }^{\circ}\right)\end{array}$ & $\begin{array}{c}\text { Wind } \\
\text { speed } \\
(\mathbf{m} / \mathbf{s})\end{array}$ & $\begin{array}{c}\text { Wave } \\
\text { period } \\
(\mathbf{s})\end{array}$ & $\begin{array}{c}\text { Wave } \\
\text { direction } \\
\left({ }^{\circ}\right)\end{array}$ & $\begin{array}{c}\text { Wave } \\
\text { height (m) }\end{array}$ \\
\hline 1 & & 60 & 10 & 7.1 & 289 & 3.3 \\
2 & VV & 90 & 10 & 7.7 & 279 & 3.5 \\
3 & & 120 & 11 & 8.3 & 270 & 3.7 \\
\hline 4 & & 60 & 11 & 8.3 & 283 & 3.9 \\
5 & $\mathrm{HH}$ & 90 & 11 & 8.3 & 283 & 3.9 \\
6 & & 120 & 12 & 8.6 & 276 & 4.0 \\
\hline \hline
\end{tabular}

\section{RESULTS}

In this section we investigate the accuracy of the theoretical models to represent the NetRAD bi-static dataset. We first show an example of the model fits and then report on the number of modes required by the $3 \mathrm{MD}$ model. Then, we study the model accuracy using the goodness of fit metrics, focusing firstly on the overall PDF fit and then the tail of the distribution.

The example in Fig. 1 shows the results for run 4 of the NetRAD bistatic data which has $\mathrm{HH}$ polarization and a bistatic angle of $60^{\circ}$. For this result, the $3 \mathrm{MD}$ model has been fitted to the data with $I=5$ modes. The parameter estimates are given in Table II, while the BD and threshold error results are given in Table III. For the KN and KR distributions we observe a strong mismatch in the body of the distribution (Fig. 1a) with high $\mathrm{BD}$ values, while the $\mathrm{PN}$ and $3 \mathrm{MD}$ models fit the data closely over all intensity values. However, the CCDF fits in Fig. 1b clearly shows that the KN and the PN distributions both have a mismatch in modelling the tail of the distribution by under and over estimating the tail respectively, while the $3 \mathrm{MD}$ and the KR both match closely. This is reflected in the threshold error values in Table III.

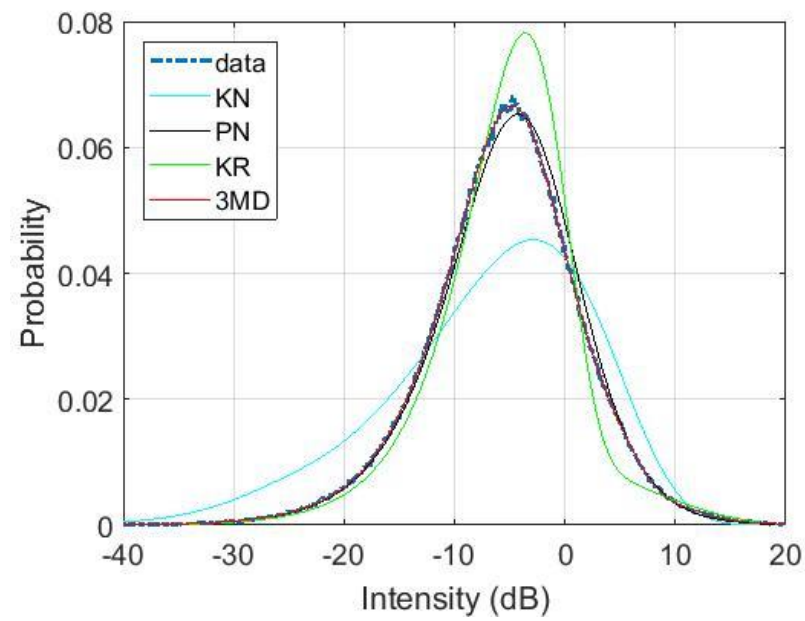

(a) PDF

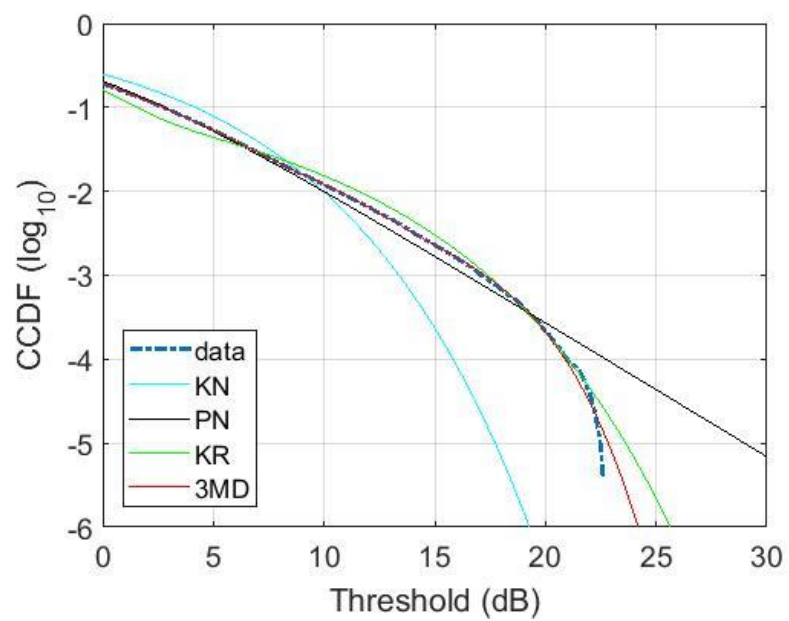

(b) $\mathrm{CCDF}$

Fig. 1. Amplitude distributions for the NetRAD data (run 4): HHpolarization, bistatic acquisition, bistatic angle $60^{\circ}$. Illustrations of (a) PDF and (b) CCDF results. 
TABLE II. PARAMETER ESTIMATES FOR THE MODELS IN FIG. 1.

\begin{tabular}{ll}
\hline CNR $(\mathrm{dB})$ & 28.9 \\
KN shape & 0.58 \\
PN shape & 1.6 \\
K+Rayleigh shape & 0.04 \\
K+Rayleigh $\mathrm{kr}-$ value & 0.41 \\
3MD mode $1(\mathrm{a}, \mathrm{c})$ & $(0.458,0.379)$ \\
3MD mode 2 $(\mathrm{a}, \mathrm{c})$ & $(0.682,0.345)$ \\
3MD mode 3 $(\mathrm{a}, \mathrm{c})$ & $(1.163,0.220)$ \\
3MD mode $4(\mathrm{a}, \mathrm{c})$ & $(2.334,0.0488)$ \\
3MD mode 5 $(\mathrm{a}, \mathrm{c})$ & $(5.463,0.0064)$ \\
\hline \hline
\end{tabular}

TABLE III. GOODNESS OF FIT METRICS FOR THE MODELS IN FIG. 1. THRESHOLD ERROR IS MEASURED AT A CCDF OF $10^{-4}$.

\begin{tabular}{cc|c}
\hline \hline & BD $(\mathrm{dB})$ & Threshold error $(\mathrm{dB})$ \\
\hline KN & -15.65 & 5.17 \\
$\mathrm{PN}$ & -31.55 & -1.73 \\
$\mathrm{KR}$ & -22.75 & -0.02 \\
3MD & -37.31 & 0.11 \\
\hline \hline
\end{tabular}

\section{A. 3MD Distribution: Number of Modes}

It has previously been reported that $I=3$ modes are sufficient to accurately model amplitude distributions of high spatial resolution sea clutter data collected by spaceborne SAR sensors [10] as well as airborne RAR and SAR instruments [11].

We now investigate how many modes of the 3MD distribution are required to accurately model the mono and bistatic sea clutter from the NetRAD dataset. The minimum number of modes required for a good fit is determined when the BD goes below the desired value of $-30 \mathrm{~dB}$. Fig. 2 shows an example model fit from the NetRAD bistatic data with $60^{\circ}$ bistatic angle and VV polarization (run 1) with different numbers of components, $I$. Table IV summarizes the minimum number of modes required by the NetRAD data. Among the 12 datasets (monostatic and bistatic), 7 need 2 or 3 modes (which is in agreement with [10] and [11]) but we find that 5 datasets need 4 or 5 modes. The link between the number of modes and the acquisition parameters are not obvious from the available data and further study is required to fully understand this relationship. Nevertheless, we have demonstrated that the $3 \mathrm{MD}$ distribution is able to accurately model the amplitude distribution of each of the NetRAD datasets, but at the cost of a greater number of parameters to estimate, $2 I$.

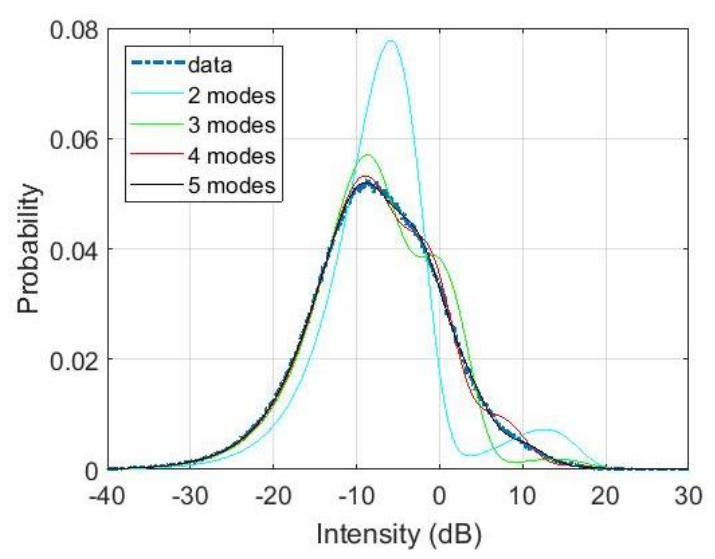

(a) PDF

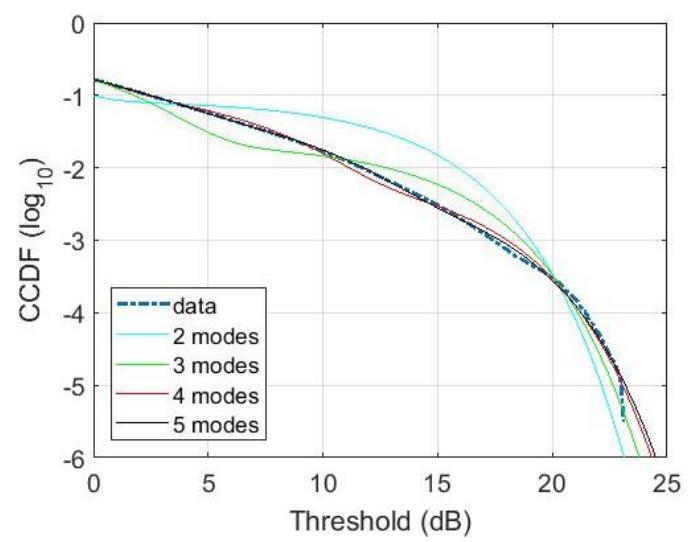

(b) $\mathrm{CCDF}$

Fig. 2. Model fits for the 3MD distribution with different number of modes, VV-polarization, bistatic configuration, $60^{\circ}$ bistatic angle (run 1).

TABLE IV. 3MD DISTRIBUTION: MINIMUM NUMBER OF MODES REQUIRED TO ACCURATELY MATCH THE NETRAD DATA.

\begin{tabular}{|c|c|c|c|c|}
\hline \multirow{3}{*}{$\begin{array}{c}\text { Bistatic } \\
\text { angle }\end{array}$} & \multicolumn{4}{|c|}{ Polarization } \\
\hline & \multicolumn{2}{|c|}{$\mathrm{HH}$} & \multicolumn{2}{|c|}{ VV } \\
\hline & Monostatic & Bistatic & Monostatic & Bistatic \\
\hline 60 & 4 & 4 & 3 & 4 \\
\hline 90 & 4 & 3 & 2 & 5 \\
\hline 120 & 3 & 3 & 2 & 3 \\
\hline
\end{tabular}

\section{B. NetRAD Radar Sea Clutter: Analysis of Model Accuracy}

In this section we study the accuracy of the four models described in Section III when fitting amplitude distributions to the NetRAD dataset. Fig. 3 first shows the BD for each bistatic angle and polarization. The only result that shows a good match for nearly every model $(\mathrm{BD} \leq-30 \mathrm{~dB})$ is the monostatic case with VV polarization (Fig. 3c). However, it is only the $3 \mathrm{MD}$ distribution that has a consistently low $\mathrm{BD}$ over all the datasets. The other results are mixed with the PN and KR models both providing a good fit with some datasets, while mismatching with others (BD > $-30 \mathrm{~dB}$ ). The $\mathrm{KN}$ model nearly always fails to accurately fit the data.

Results of the threshold error for a CCDF of $10^{-4}$ are then displayed in Fig. 4, where low errors are observed for both the 
$\mathrm{KR}$ and $3 \mathrm{MD}$ models $(< \pm 1 \mathrm{~dB})$, while the $\mathrm{PN}$ and $\mathrm{KN}$ distributions have a greater mismatch with errors up to $\pm 4 \mathrm{~dB}$. If the PN distribution was used in a detection scheme with such a large mismatch, there will be a large number of missed detections. Conversely, if the KN distribution were used, there would be a higher probability of false alarm.
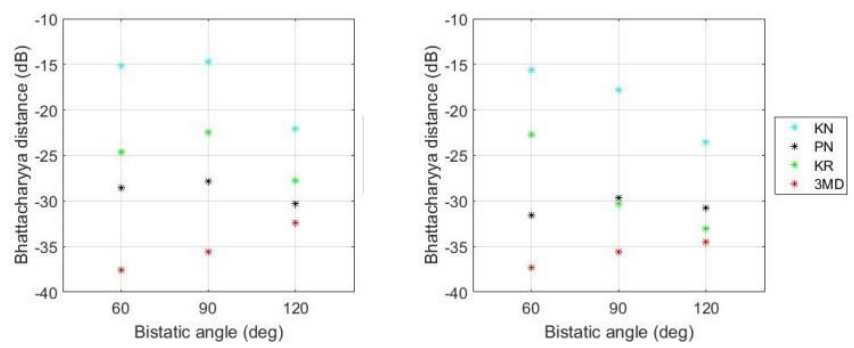

(a) $\mathrm{HH}$ pol., monostatic

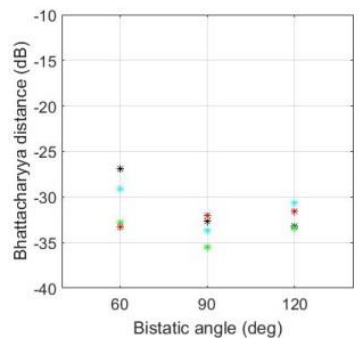

(c) VV pol., monostatic

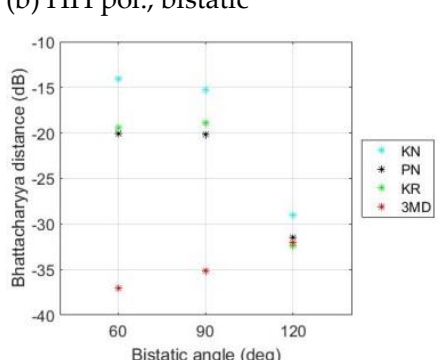

(d) VV pol., bistatic

Fig. 3. Bhattacharyya distance (in $\mathrm{dBs}$ ).
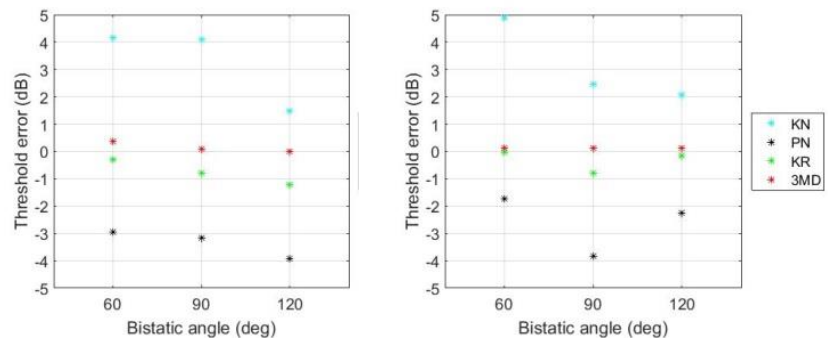

(a) $\mathrm{HH}$ pol., monostatic

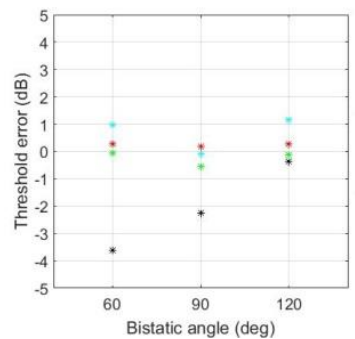

(b) $\mathrm{HH}$ pol., bistatic

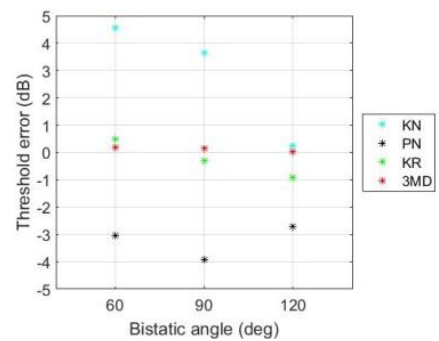

(d) VV pol., bistatic

match in the tail region, making them a good choice. When considering the total fit to both the body and distribution tail, only the 3MD model is able to consistently provide good results, but at the cost of a higher number of parameters to be estimated.

\section{ACKNOWLEDGMENT}

Part of this work is funded by the French Ministry of Armed Forces (DGA - Direction Générale de l'Armement) under the COMAREM project run by the French Defense Procurement Agency. Research presented herein was also carried out in part under the NATO SET-185 program.

\section{REFERENCES}

[1] L. Rosenberg, "Sea-Spike Detection in High Grazing Angle X-Band Sea-Clutter," in IEEE Transactions on Geoscience and Remote Sensing, vol. 51, no. 8, pp. 4556-4562, Aug. 2013.

[2] H. D. Griffiths, W. A. Al-Ashwal, K. D. Ward, R. J. A. Tough, C. J. Baker, and K. Woodbridge, "Measurement and modeling of bistatic radar sea clutter", in IET Radar, Sonar and Navigation, vol. 4, no. 2, pp. 280-292, 2010.

[3] E. Jakeman and P. Pusey, "A model for non-Rayleigh sea echo," in IEEE Transactions on Antennas and Propagation, vol. 24, no. 6, pp. 806-814, November 1976.

[4] K. D. Ward, R. J. A. Tough, and S. Watts, "Sea Clutter: Scattering, the K-Distribution and Radar Performance", 2nd ed. Herts, U.K.: The Institute of Engineering Technology, 2013.

[5] A. Fiche, S. Angelliaume, L. Rosenberg and A. Khenchaf, "Analysis of X-Band SAR Sea-Clutter Distributions at Different Grazing Angles," in IEEE Transactions on Geoscience and Remote Sensing, vol. 53, no. 8, pp. 4650-4660, Aug. 2015.

[6] G. V. Weinberg, "Assessing Pareto fit to high-resolution high-grazingangle sea clutter," in Electronics Letters, vol. 47, no. 8, pp. $516-517,14$ April 2011.

[7] L. Rosenberg and S. Bocquet, "Application of the Pareto Plus Noise Distribution to Medium Grazing Angle Sea-Clutter," in IEEE Journal of Selected Topics in Applied Earth Observations and Remote Sensing, vol. 8, no. 1, pp. 255-261, Jan. 2015.

[8] W. A. Al-Ashwal, Woodbridge, K. and H. D. Griffiths, "Analysis of Bistatic Sea Clutter - Part II: Amplitude Statistics", in Transactions on Aerospace and Electronic Systems, vol. 50, no. 2, April 2014.

[9] T. Lamont-Smith, "Translation to the normal distribution for radar clutter," in IEE Proceedings - Radar, Sonar and Navigation, vol. 147, no. 1, pp. 17-22, Feb. 2000.

[10] C. H. Gierull and I. Sikaneta, "A Compound-Plus-Noise Model for Improved Vessel Detection in Non-Gaussian SAR Imagery," in IEEE Transactions on Geoscience and Remote Sensing, vol. 56, no. 3, pp. 1444-1453, March 2018.

[11] L. Rosenberg and S. Angelliaume, "Characterisation of the tri-modal discrete sea clutter model", in IEEE International Radar Conference, pp. 1-6, 2018.

[12] L. Rosenberg and S. Bocquet, "Non-coherent Radar Detection Performance in Medium Grazing Angle X-Band Sea Clutter," in IEEE Transactions on Aerospace and Electronic Systems, vol. 53, no. 2, pp. 669-682, April 2017.

[13] S. Bocquet, "Parameter estimation for Pareto and K distributed clutter with noise," in IET Radar, Sonar \& Navigation, vol. 9, no. 1, pp. 104113, 12015.

[14] L. Rosenberg, S. Watts and S. Bocquet, "Application of the K+Rayleigh distribution to high grazing angle sea-clutter," in IEEE International Radar Conference, pp. 1-6, 2014.

[15] M. Ritchie, A. Stove, K. Woodbridge and H. Griffiths, "NetRAD: Monostatic and Bistatic Sea Clutter Texture and Doppler Spectra Characterization at S-Band," in IEEE Transactions on Geoscience and Remote Sensing, vol. 54, no. 9, pp. 5533-5543, Sept. 2016. 